\title{
Dispositivos Biopolíticos de Integración de la Exclusión Social: El Negocio de la Educación de los Pobres
}

\author{
Biopolitical Devices of Social Integration: The Business of \\ Education for the Poor
}

\author{
Juan Ramón Rodríguez Fernández * \\ Enrique Javier Díez Gutiérrez \\ Universidad de León, España
}

\begin{abstract}
Los programas de ingresos mínimos son actuaciones públicas esenciales en la lucha contra la pobreza en la Unión Europa. En este artículo pretendemos mostrar las características de las políticas de ingresos mínimos de lucha contra la pobreza y de las actuaciones formativas vinculadas a ellas en el caso de España. Partiendo de una estrategia analítica basada en los planteamientos de Michel Foucault, analizamos los discursos hegemónicos en estos programas de ingresos mínimos: el discurso socialliberal y el discurso neoliberal. Para ello utilizamos las nociones de racionalidad política y de la sintomatología social. A través de este análisis discursivo encontramos que, en las actuaciones de lucha contra la exclusión social, la educación se configura como una inversión individual para el fomento de la empleabilidad, como un elemento para reivindicar la responsabilidad individual del sujeto en su propio proceso de integración social y como un espacio susceptible de rentabilidad económica mediante su configuración como un cuasimercado educativo. A su vez, y este es uno de los principales resultados de este trabajo, se identifica un emergente discurso hegemónico en la lucha contra la pobreza. Un discurso fruto de la hibridación de los discursos social y neoliberal, que hemos denominado Educación como "santa neocruzada" contra la pobreza.
\end{abstract}

Descriptores: Política; Discurso; Justicia social; Exclusión social; Empresa.

\begin{abstract}
The minimum income programs are essential public actions in the fight against poverty in the European Union. In this article, we intend to show the characteristics of the minimum income policies for the fight against poverty and the training activities linked to them in the case of Spain. Starting from an analytical strategy based on the approaches of Michel Foucault, we analyze the hegemonic discourses in these minimum income programs: the social-liberal discourse and the neoliberal discourse. For this we use the notions of political rationality and the social symptomatology. Through this discursive analysis we find that, in the actions of struggle against social exclusion, education is configured as an individual investment for the promotion of employability, as an element to claim the individual responsibility of the subject in its own integration process social and as a space susceptible of economic profitability through its configuration as an educational quasi-market. In turn, and this is one of the main results of this work, an emergent hegemonic discourse is identified in the fight against poverty. A discourse resulting from the hybridization of social and neoliberal discourses, which we have called Education as a "neo-crippled saint" against poverty.
\end{abstract}

Keywords: Politics; Speeches; Social justice; Social exclusion; Enterprises.

*Contacto: jrodrf@unileon.es

ISSN: 2254-3139

www.rinace.net/riejs/

revistas.uam.es/riejs
Recibido: $\quad 7$ de marzo 2018

$1^{\text {a }}$ Evaluación: 1 de julio 2018

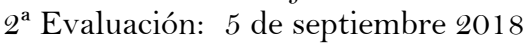

Aceptado: 29 de septiembre 2018 


\section{Las políticas sociales de lucha contra la exclusión social: Las rentas mínimas de inserción}

Los principales programas socioeducativos de lucha contra la pobreza en los países de la Unión Europea son las RMI-Rentas Mínimas de Inserción. Estos programas surgen a principios de la década de los años 90 mediante la Recomendación del Consejo 92/441 de 24 de junio de 1992, que insta a los países miembros de la UE-Unión Europea a desarrollar programas que garanticen unos ingresos mínimos a su ciudadanía.

A lo largo de la década de los años 90 y principios del siglo XXI los diferentes países de la UE han ido desarrollando este tipo de programas, con una gran heterogeneidad en términos de cuantía económica, requisitos, duración, etc. (Malgesini, 2017). Siendo sus destinatarios aquellas personas con ingresos inferiores al umbral de la pobreza, para cuyo indicador se toma como referencia el $60 \%$ de la mediana de la distribución de los ingresos en una determinada zona, región o país. Según este estadístico, en la UE en el 2016 había un $23 \%$ de la población por debajo de este umbral, el país con la tasa más alta de pobreza sería Rumanía con un 38,8\% y el país con la menor Islandia con apenas un 13\%. Mientras que España rondaríamos el 28\% (EUROSTAT, 2018).

A pesar de la gran heterogeneidad existente en estos programas de ingresos mínimos, tanto en el contexto europeo como en el caso concreto español (Frazer y Marlier, 2016), podemos señalar la existencia de una doble estructura común: a) un ingreso económico con carácter periódico. Y, b) la vinculación de la cuantía económica con la realización de diversas actividades dirigidas a la inserción social y laboral de la persona beneficiaria de la prestación económica. Actividades que se desarrollan en diversas áreas (familiar, personal, salud, educativa, laboral...) a través de un Itinerario Personalizado de Inserción, el cual incluye diversos compromisos consensuados con el titular de la prestación para participar y realizar las actividades propuestas por los profesionales de los servicios sociales. Entre las que destacan especialmente la realización de cursos de formación para el fomento de la empleabilidad y la activación laboral.

\subsection{Las limitaciones de las rentas mínimas de inserción}

Es en el contexto del Modelo Social Europeo asentado en sistemas públicos de bienestar social (Esping-Andersen, 2000) en el que surgen originalmente las RMI, como propuestas socialdemócratas destinadas a favorecer la integración de los sectores marginales y empobrecidos en la sociedad. Sin embargo, a pesar de tener entre sus objetivos la lucha contra la pobreza, el aumento de la cohesión social y la protección social de la ciudadanía, han tenido una serie de limitaciones y problemas inherentes a su propia naturaleza. Limitaciones que han sido acrecentadas por el influjo y las transformaciones que el discurso hegemónico neoliberal está operando en estos programas (Díez Gutiérrez, 2007). Transformaciones que pueden entenderse bajo el desplazamiento que ha ocurrido desde los planteamientos del welfare, basados en los derechos sociales universales de ciudadanía, a el workfare, en el cual se supedita estos derechos sociales al paso e inserción activa en el mercado laboral.

Esta evolución puede verse reflejada en algunas de las siguientes características de las RMI: En primer lugar, es necesario apuntar la escasa cobertura social que ofrecen estas 
medidas. La cuantía media de las RMI en España en el año 2015 era de 435,80€ (Ministerio de Servicios Sociales, 2015), cuantía insuficiente para cubrir gastos básicos como los de alojamiento y manutención, solamente el precio medio de una vivienda en alquiler en España supera esa cuantía. En el año 2012, en los años más duros de la crisis capitalista del 2008, únicamente se dedicaba un 0,49\% del presupuesto de las comunidades autónomas españolas a la financiación de las RMI, siendo el País Vasco la comunidad con mayor porcentaje dedicado a las RMI (3,28\%) y Extremadura la que menos dedicaba con un 0,03\% de gasto público (Arriba González de Durana, 2014). El módulo básico de la prestación económica se sitúa siempre por debajo del umbral de la pobreza, con lo que podemos afirmar que las políticas sociales para pobres, sobre todo en estos tiempos de emergencia social y de austeridad neoliberal, son políticas sociales pobres.

Otra cuestión crítica en estos programas de RMI es el carácter estigmatizador y burocrático que tienen al focalizar su atención en el colectivo de personas pobres y no dirigirse al conjunto de la ciudadanía. La puesta en marcha de estos programas de ingresos mínimos implica la creación de todo un entramado burocrático y administrativo impulsado por la aparición de nuevas tecnologías y aplicaciones informáticas que permiten controlar y cruzar datos de los usuarios con mayor precisión- dedicado a la evaluación, supervisión y control de las condiciones económicas, familiares y sociales de las personas que solicitan estas prestaciones (Raventos, 1999). Una evaluación dirigida a la comprobación de recursos y de requisitos exigidos para el acceso a este derecho que favorece la burocratización de las tareas de los profesionales, en detrimento de un trabajo verdaderamente educativo con los usuarios de esta prestación. A su vez, es un proceso que abre además las puertas a posibles intromisiones por parte de la administración pública en la vida y circunstancias privadas de las personas solicitantes, que genera lentitud y retrasos en la tramitación de estas ayudas y que por su naturaleza (lenguaje, formato, etc.) actúa como batería de trabas y barreras burocráticas ante los colectivos más desfavorecidos (Oliver, 2013).

Otro aspecto es su tendencia a considerar la pobreza desde un punto de vista individual, cuando se trata de una problemática cuyo origen aparece fundamentalmente en el plano social. La puesta en marcha de un itinerario individualizado de inserción en el que se trabajen aquellas áreas de la persona con déficits y carencias que dificulten su integración social y laboral contribuye a reforzar la idea de que la exclusión social es una problemática de la propia persona, de sus características y de sus carencias. Al enfatizar la búsqueda de soluciones en el plano individual, se tiende a soslayar el carácter político y estructural que tiene la pobreza y la desigualdad social (Bauman, 2004).

Por último, desde el Tratado de Lisboa del año 2000, las políticas europeas de lucha contra la exclusión se han ido sustentando cada vez más en esta idea de la activación individual a través del fomento de la empleabilidad y del emprendimiento. Para lo cual, la educación y la formación desempeñan un papel central como elementos impulsores de la inserción laboral y del crecimiento económico. Como la propia Comisión Europea (2010) señala en el documento Estrategia Europa 2020: “...un mejor nivel educativo ayuda a encontrar trabajo y los avances en el aumento de la tasa de empleo ayudan a reducir la pobreza”.

Siguiendo a Michel Foucault (2009), son dispositivos biopolíticos dirigidos a la gestión gubernamental de determinados colectivos de la población. Instrumentos que integran y hacen más fácilmente asumible para la opinión pública el problema social de la marginalidad y la exclusión. 


\section{Análisis político del discurso educativo en los programas de ingresos mínimos}

Las actuaciones de formación son una de las medidas más importantes dentro de los itinerarios de inserción desarrollados en los programas de RMI a la hora de fomentar la inserción de las personas en riesgo de exclusión. En este sentido, es posible señalar que las acciones de formación insertas en las RMI y dirigidas a colectivos en riesgo social, tienen una serie de características y asumen unos principios cada vez más influenciados por el pensamiento hegemónico neoliberal (Gómez Sánchez, Navas Saurín y Bernard García, 2005; Rodríguez Fernández, 2013).

El proceso de implementación de las diferentes políticas sociales y educativas en todo el mundo, debe ser entendido como resultado de la extremadamente compleja interacción entre dos racionalidades políticas hegemónicas en nuestras sociedades ${ }^{1}$. Por un lado, estaría la racionalidad política social-liberal o ilustrada, heredera de los principios filosóficos de la Ilustración, hoy en día está en claro retroceso, pero que aún conserva vigor y representación en las visiones y discursos actuales con sus planteamientos sobre la equidad, la justicia social y el papel privilegiado de la educación como motor del desarrollo personal y social dentro del marco establecido por un capitalismo regulado por el Estado?; y por otra parte la racionalidad política neoliberal, en la actualidad claramente dominante y que se asienta en los principios del libre mercado, la libertad individual, la competencia y el crecimiento económico. Es en el debate hegemónico entre estas dos concepciones donde es posible entender buena parte de las transformaciones que estamos presenciando en las políticas educativas y sociales, y en concreto en las políticas socioeducativas de lucha contra la exclusión, como los programas de ingresos mínimos.

Siguiendo a autores como Michel Foucault (2009), Ernesto Laclau y Chantal Muffe (1987) o Rosa Buenfil Burgos (1996) la interacción dinámica entre estas dos racionalidades políticas, interacción que puede asumir diversas formas: cooperación, oposición, adaptación mutua, etc., en un determinado campo genera una serie de dispositivos gubernamentales -los programas de rentas mínimas de inserción se pueden considerar como ejemplos de estos dispositivos-, y da lugar a una serie de efectos sociales. Este imaginario social está siendo constantemente disputado por otros discursos alternativos con racionalidades políticas y tecnologías de gobierno diferentes, que en ocasiones se oponen frontalmente a los discursos hegemónicos y en otras ocasiones son asumidas e incorporadas por el discurso dominante en una suerte de hibridación de discursos.

El conjunto inseparable e interrelacionado que forman las racionalidades políticas, los dispositivos biopolíticos y sus efectos sociales es lo que podemos denominar discurso, entendido no como una mera alocución o producción escrita, sino como una práctica social e histórica con efectos performativos, tanto en plano social, como en los procesos de subjetivación a través de los cuales nos construimos como personas (Gómez Sánchez, Navas Saurín y Bernard García, 2005).

\footnotetext{
${ }^{1}$ Y no solamente a través de los discursos hegemónicos, sino también a través de las diversas interpretaciones que los sujetos hacemos a la hora de ponerlas en práctica en contextos singulares, históricos y concretos, es decir, cómo las transformamos cuando las convertimos en prácticas, actuaciones, expresiones o modos de comportamiento, etc. (Ball, 2012). ${ }^{2}$ En la actualidad uno de los más destacados defensores de esta racionalidad es el psicólogo social Steven Pinker (2018).
} 
En el ámbito de las actuaciones formativas en los programas de ingresos mínimos para colectivos en riesgo de exclusión social encontramos la siguiente sintomatología, que actualmente está siendo fuertemente cuestionada debido a los problemas que tiene a la hora de dar respuesta a los problemas de nuestras sociedades.

\subsection{La educación como elemento de inversión individual y progreso social}

La educación en los programas de rentas mínimas de inserción se apoya en los presupuestos económicos de la Teoría del capital humano (Becker, 1985). Para esta teoría, la educación es una inversión de carácter individual con valor de cambio en el mercado de trabajo (Schultz, 1962). Para esta teoría la educación no solamente aumenta la empleabilidad de la persona, sino que es un elemento directo a la hora de mejorar la productividad, el crecimiento económico y, de esa manera, aumentar la calidad y el volumen de los puestos de trabajo disponibles. Desde este planteamiento, y como señalan las principales estrategias y directrices europeas en la lucha contra la pobreza y la exclusión, las personas pobres tendrían que formarse y adquirir conocimientos, habilidades, competencias requeridas por el sistema productivo, que les permitieran aumentar su empleabilidad y así poder insertarse efectivamente en el mercado de trabajo (Muñoz de Bustillo y Bonete, 2009). La cuestión central para fomentar la inserción social y laboral de estas personas, sería la de formar en las competencias profesionales más demandadas por el sistema productivo.

Sin embargo, estos planteamientos tienen serias dificultades para cumplir sus objetivos. En primer lugar, es cuestionable la existencia de una relación directa y mecánica entre formación, inserción laboral, aumento de la productividad y mejora de las condiciones laborales como resultado del crecimiento económico que generan los y las trabajadoras mejor formadas (Chang, 2012). Hoy en día nuestras sociedades tienen la juventud más y mejor formada y no por ello el desempleo o la precariedad se han visto reducidos en los últimos años. Las condiciones de trabajo y el volumen de puestos de trabajo de un país o de una determinada zona no dependen de los niveles de formación alcanzados o del sistema educativo, sino de la relación de fuerzas entre el capital y el trabajo, equilibrio que se ha visto radicalmente alterado a favor del primero mediante las políticas económicas neoliberales y especialmente desde la crisis del 2008 (Harvey, 2012).

En segundo lugar, la educación asume un rol pasivo dentro del subsistema laboral, dedicada únicamente a formar en las competencias requeridas por el mercado. Así, la educación es vista en sí misma, en todos los niveles del sistema educativo, ya sea educación primaria o secundaria, ya sea educación formal o no formal, como un elemento cuya función principal es la preparación y la competición para el mundo laboral. Desde este punto de vista, la metodología didáctica que resulta más funcional a estos planteamientos es el modelo por competencias, modelo que ha colonizado los planes de estudio en todos los niveles educativos y las prácticas y subjetividades del profesorado. Este modelo parte de la asunción de que es posible parcelar en diferentes técnicas una determinada profesión, pudiendo derivar posteriormente acciones formativas concretas para cada competencia o técnica. Se trata de una concepción simplista de la esfera profesional, en la cual ésta se ve reducida a un mero stock de competencias profesionales y al trabajador como un mero técnico. Con esta concepción técnica, se pierden de referencia los factores éticos, sociales $\mathrm{y}$ culturales que afectan a las profesionales y se ofrece una imagen artificial y simplificadora de la complejidad de las tareas y procesos todo trabajador pone en marcha 
en su trabajo, los cuales no pueden reducirse meramente al conocimiento y puesta en marcha de competencias profesionales (Schön, 1992).

En tercer lugar, la educación pasa a ser considerada como un producto de consumo, con un valor de cambio en el mercado laboral que aumenta la empleabilidad de la persona que lo adquiere, en la competición con otras personas dentro del mercado de trabajo. Esta visión contribuye a favorecer el consumismo en educación y la organización de los sistemas educativos según los principios del mercado y de la libre competencia. La idea de educación a lo largo de la vida contribuye a reforzar ese consumismo, en el que todos y todas estaremos abocados a consumir educación durante toda la vida para poder seguir siendo personas productivas y empleables, asumiendo personalmente los gastos de esa formación "a lo largo de toda la vida". Configurando así la educación como un nicho de negocio privado más, similar en términos de explotación económica al de la empresa internacional del automóvil (Hill, 2013).

Por último, la Teoría del capital humano en educación ofrece una visión meramente técnica y meritocrática de la relación entre empleado y empleador, sin considerar los aspectos ideológicos y estructurales que condicionan las condiciones de contratación laborales en los puestos de trabajo (Bowles y Gintis, 1983). Esta teoría que fundamenta las políticas socioeducativas de lucha contra la pobreza, otorga a la educación un rol central, al considerar que es el elemento central a la hora de favorecer la inserción sociolaboral del sujeto. Así, la pobreza y la desigualdad social son problemáticas sociales que se pueden solucionar, simplemente, con más educación y sobre todo con una educación más estrechamente vinculada al mundo del trabajo. Desde esta visión, el desempleo y la pobreza son problemas cuyo origen está o bien en que la educación no formal de manera adecuada en función de los requerimientos y demandas del mercado (como si ese fuera el objetivo de la educación), que los docentes no forman con las metodologías didácticas adecuadas al modelo de gestión empresarial para el que se quiere "entrenar" (como si esa fuera la metodología adecuada), o bien en que la persona en situación de exclusión social o en condiciones laborales precarias no ha sabido aprovechar las oportunidades formativas, educativas, laborales... que ha tenido a lo largo de su vida (la culpa de su situación y de su pobreza acaba siendo de la propia víctima, re-victimizándola).

\subsection{La educación como redención del pobre}

En el marco de los programas de ingresos mínimos, las personas pobres se caracterizan por tener una serie de lagunas y carencias que suponen barreras para su integración social, muy especialmente su integración activa en el mundo laboral Así, las acciones formativas insertas en los itinerarios de incorporación social deben orientarse al fomento de las habilidades profesionales para la empleabilidad. Desde esta perspectiva son actuaciones formativas habituales las siguientes: cursos de formación pre-laboral, cursos de formación específica, cursos de cómo elaborar un currículum vitae, cómo afrontar las entrevistas de trabajo, cómo buscar empleo por Internet, cursos sobre cómo emprender y crear tu propio negocio y un largo etcétera de acciones que se orienten a favorecer la empleabilidad de estos colectivos. En esta misma línea se sitúan las medidas dirigidas a dotar de habilidades sociales y actitudinales: mejora de la autoestima, habilidades personales, inteligencia emocional, higiene personal, organización y economía doméstica, etc. En un contexto laboral donde el trabajo es escaso y precario (Standing, 2013) se enfatiza la inserción social de estos colectivos fundamentalmente a través de la inserción en el mundo del trabajo, mediante la adquisición de competencias profesionales de bajo rango y habilidades sociales 
y actitudinales. Porque, como todos sabemos, las personas pobres lo que necesitan son cursos de autoestima porque suelen estar deprimidos, de economía doméstica, porque no saben llevar las cuentas de un hogar y de inteligencia emocional porque no saben cómo procesar emocionalmente su situación de precariedad o exclusión social.

Aunque las acciones formativas en los programas de ingresos mínimos tienen como objetivo formal mejorar la empleabilidad y favorecer la participación en el mercado laboral. Sin embargo, cumplen sobre todo una función diferente y es la actuar como elemento redentor del pobre. Participando en estos cursos formativas las personas en exclusión social han de demostrar fehacientemente a la sociedad su buena predisposición a insertarse en las condiciones y normas establecidas por la propia sociedad que les excluyó previamente. La participación en la formación actúa como un mecanismo que permite diferenciar aquellos pobres que se esfuerzan y que merecen recibir la ayuda económica, quienes muestran en términos neocatólicos: "contrición, arrepentimiento, dolor de sus pecados, propósito de la enmienda y cumplir la penitencia”. Lo cual les diferencia claramente de aquellos otros pobres que no demuestran esforzarse lo suficiente (no muestran suficiente arrepentimiento, dolor de sus pecados y propósito de la enmienda) y que, por lo tanto, no son merecedores del "perdón” y la prestación económica. Es una manera de volver a las concepciones medievales sobre la caridad, los pobres verdaderos y los pobres fingidos. El derecho de ciudadanía social (Marshall, 1950) por el cual todo ciudadano y ciudadana tiene derecho a un mínimo de subsistencia, deja de ser un derecho reconocido, para pasar a ser algo que hay que ganarse mediante la predisposición a participar en itinerarios de inserción y mediante la realización de acciones de formación.

\subsection{Las acciones formativas para personas pobres como forma de alienación}

La educación bajo estas propuestas es dudosa que pueda ser considerada un instrumento que facilite la igualdad social, ni mucho menos que se oriente a la emancipación social y a la búsqueda de condiciones que fomenten la justicia social. Las acciones formativas que se sitúan dentro de los programas de ingresos mínimos en el territorio español, pero también en toda Europa desde el Tratado de Lisboa de 2000 con el desarrollo de políticas sociales de activación (Fernández et al., 2014), contribuyen a la alienación de las personas pobres mediante la inculcación de las siguientes nociones como si fuesen ideas de sentido común.

En primer lugar, se acepta la idea por la cual mayores niveles educativos de la ciudadanía implican mayores cotas de igualdad social. La esencia de las acciones formativas insertas en los programas de ingresos mínimos se asienta en la lógica por la cual la educación es siempre buena per se y que la inmensa mayoría de los problemas sociales pueden ser solventados si conseguimos extender la educación a todas las capas sociales. Desde este punto de vista, la solución para buena parte de los problemas sociales (desempleo y pobreza, pero también racismo, violencia de género, etc.) sería únicamente el desarrollo de acciones formativas especialmente diseñadas para solucionar tales problemáticas, no siendo necesarias otro tipo de actuaciones estructurales para remover las causas profundas en las que se originan estas problemáticas. Como planteaba el ex primer ministro inglés Tony Blair: "Educación, educación y más educación” (Wrigley, 2005). De esta forma, la precariedad y el desempleo son problemas achacables fundamentalmente a la educación, que no forma adecuadamente, o a las propias personas que no tienen las competencias profesionales requeridas, las actitudes más adecuadas o que no se esfuerzan (emprenden) lo suficiente o a los propios formadores y educadores que no enseñan siguiendo adecuadamente el modelo por competencias. Sin embargo, es necesario apuntar que, 
aunque haya mayores niveles formativos en el conjunto de la ciudadanía, o la educación sea de más calidad o se extienda a todas las capas de la sociedad, no necesariamente nuestras sociedades van a ser más justas como plantea la racionalidad ilustrada.

En segundo lugar, estas propuestas formativas contribuyen a reforzar el predominio de los valores de la competitividad y el individualismo exacerbado como valores nucleares para el progreso del individuo y el desarrollo social según el pensamiento neoliberal. La pertenencia social es una cuestión individual de adquisición de competencias o habilidades profesionales, donde las personas compiten entre sí con sus credenciales formativas por los puestos de trabajo. Así, se asume la noción del hombre empresa (Foucault, 2009) por la cual el sujeto se autorrealiza en la libre competición con los demás mediante la inversión en sí mismo, en este caso en capital humano.

$\mathrm{Al}$ enfatizar el carácter individual del proceso de inserción social, y al soslayar las bases estructurales de la pobreza y el desempleo, se subraya la responsabilidad individual del propio sujeto en su situación social -si eres pobre o tienes un trabajo precario es porque no te has esforzado lo bastante o no has aprovechado las oportunidades que la sociedad le ha ofrecido-. Así, se abre la puerta a la culpabilización y al tratamiento punitivo de la marginalidad social (Wacquant, 2010), las personas pobres son pobres porque son vagas, porque no se esfuerzan lo bastante, algunas de ellas son delincuentes... y por ello, el estado público tiene que reducir o eliminar las ayudas dirigidas a estos colectivos y/o en todo caso sustituirlas por mecanismos punitivos y de control social. Como decía Mitt Romnney el candidato a la presidencia de los Estados Unidos en el 2012 en plena campaña electoral: "Mi trabajo no consiste en preocuparme por esas personas (en referencia a los trabajadores pobres y a los beneficiarios de ayudas sociales). Nunca les convenceré de que deben asumir sus responsabilidades personales y ocuparse de sus vidas" (Krugman, 2012). Esta noción del pobre, del excluido, es la cara reversa del modelo neoliberal actual de éxito social: el emprendedor aquella persona que con su esfuerzo individual y en lucha y competición con sus congéneres, ha sabido interpretar y aprovechar las oportunidades que el mercado le ha dado para lograr el éxito.

En tercer lugar, se ahonda en la mercantilización de la educación y en el negocio de la pobreza. La formación para las personas beneficiarias de rentas mínimas, al igual que otros servicios públicos, es vista por parte del pensamiento neoliberal como una mercancía con posibilidades de generar beneficios económicos, a través de la financiación pública de entidades privadas encargadas de dar la formación requerida a estos colectivos. Sin duda alguna no es un nicho de mercado tan lucrativo como lo puedan ser las pensiones públicas, la atención residencial a las personas mayores, o la educación superior. Sin embargo, sí tiene el suficiente interés como para que diversas entidades y fundaciones acudan al negocio de la pobreza, a través de la organización de las acciones formativas organizadas mediante sistemas de cuasimercado (Whitty, Power y Halpin, 1999). En este marco, las instituciones públicas se encargan de planificar y organizar las acciones formativas, las cuales son posteriormente realizadas por entidades del tercer sector, fundaciones de formación, empresas, sindicatos, etc. mediante convenios de financiación pública.

\subsection{Hacia una Santa NeoCruzada por la educación de los pobres}

En los últimos años, hemos sido testigos de la compleja construcción de un nuevo discurso educativo, basado tanto en el discurso humanista como en el discurso neoliberal. Es un nuevo discurso que los autores hemos denominado una Santa NeoCruzada por la 
Educación de los pobres y que proviene de la interacción entre la racionalidad ilustrada y las racionalidades neoliberales dominantes (Rodríguez Fernández, 2016).

En este nuevo discurso, la educación es vista como algo bueno per se, como herramienta clave para combatir y erradicar la pobreza, el desempleo y casi todos los problemas sociales. Y al mismo tiempo como una oportunidad de negocio en donde cada servicio público debe ser gestionado mediante lógicas de beneficio económico a través de la privatización. Desde la atención sanitaria a la educación superior, desde las pensiones sociales a los servicios sociales. Incluso la atención social para los pobres puede, y debe ser, ser un nicho rentable.

Este discurso tiene una extraordinaria posición hegemónica porque une dos fuertes discursos que dominan el debate social ideológico actual. Por un lado, el viejo discurso humanista que todavía considera la responsabilidad social del gobierno para sus ciudadanos menos afortunados y por esa razón considera la educación como una herramienta fundamental para resolver los problemas sociales. Pero, al mismo tiempo, con un lado potencialmente paternalista y caritativo, donde la educación actúa como redención y "penitencia” para los pobres.

Pero, por otro lado, está el discurso neoliberal que considera que todo, incluido la educación debe ser considerado una mercancía a través de la cual conseguir rentabilidad y crecimiento económico. Un discurso que a su vez enfatiza la estigmatización y la responsabilidad individual del sujeto.

Estas dos grandes concepciones, han dado lugar en el campo de la atención social a una Santa NeoCruzada por la Educación del pobre. Por un lado, es "santa" porque siguiendo el viejo discurso humanista, la educación siempre es buena, una herramienta mágica para resolver todos los problemas sociales. La educación es lo que nos hace ciudadanos responsables, está en la base del pensamiento humanista ilustrado (Pinker, 2018). Y es que, ¿quién puede estar en contra de la educación y de su extensión a mayores sectores sociales? Una idea que la UE ha asumido mediante la expresión "Aprendizaje a lo largo de la vida". Pero, por otra parte, es una "cruzada", porque al mismo tiempo y siguiendo los preceptos neoliberales, la educación -incluso para pobres- contribuye a la creación de espacios en los cuales el capital privado pueda desarrollar actividades lucrativas y de negocio privado. El formato más habitual para ello es el de los cuasimercados (Apple, 2013), en donde el estado financia económicamente a entidades privadas para que estas ejecuten y pongan en marcha actividades, bajo la supervisión administrativa de las administraciones públicas.

Esta hibridación entre discursos hegemónicos es la que hace que la idea de la Santa NeoCruzada por la por la educación sea tan potente. Para mucha gente porque asume los principios neoliberales de la libre competencia, de la privatización de lo público como la mejor forma de gestión, de la inversión en uno mismo y del crecimiento económico; y, por otra parte, para otra mucha gente porque sigue los principios humanistas de la educación como panacea universal a los problemas sociales. Panacea que no requiere un análisis problemático de las cuestiones estructurales que generan tales problemas sociales.

Sin embargo, ambos discursos que fundamentan esta Santa NeoCruzada deben ser problematizados: En primer lugar, porque la privatización de la educación pública no contribuye necesariamente a mayores niveles de igualdad ni de cohesión social, al revés favorece la generación de guetos sociales (Apple, 2002); y en segundo lugar, porque la 
educación pública y estatal en sí misma es un poderoso aparato de reproducción ideológica y legitimación del orden social (Althusser, 2004), solamente una educación pública que sea contrahegemónica, es decir, que contribuya a través de la relación educativa a desvelar los mecanismos de opresión y de legitimación puede contribuir verdaderamente a la justicia social (Apple, 2013).

\section{Dislocación de discursos: La renta básica}

En este último apartado vamos a proponer un camino diferente al marcado por las políticas tradicionales de los Estados de bienestar social. Un camino fundamentado en racionalidades políticas y discursos alternativos a los hegemónicos actualmente. Se trata de discursos sociales marginados, no porque la historia haya demostrado que no son válidos a la hora de ofrecer respuestas a las necesidades y problemas sociales, sino porque han sido marginados por otros discursos en la disputa por la hegemonía a la hora de dar respuestas y sentido al mundo que nos rodea. Entre otros, los estudios de Thomas Piketty (2014) y de David Harvey (2013) muestran las dificultades del pensamiento liberal, en su forma neoliberal, a la hora de proporcionar las bases para sociedades más justas e igualitarias. Por ello, es preciso buscar propuestas y medidas asentadas en otro tipo de concepciones.

Una de estas propuestas se trata de la propuesta de la Renta Básica. Bajo esta propuesta la educación rompe con los principios neoliberales del Capital humano y de la empleabilidad, abriéndose a otras concepciones y prácticas educativas orientadas a la consecución de ciudadanos y ciudadanas capaces de entender críticamente los problemas actuales de nuestras sociedades.

\subsection{La renta básica de ciudadanía (RB)}

La RB es una propuesta que va más allá de las RMI. No se limita a abordar asistencialmente la pobreza, sino que es en sí misma una herramienta dirigida a la transformación social, la cual surge de las contradicciones y problemáticas que genera el sistema capitalista: desempleo, pobreza, marginación, precarización de las condiciones laborales, etc. A su vez, para algunos autores como Ryan Avent (2017) es una medida que tiene su justificación en la creciente automatización y robotización de puestos de trabajo en el mercado laboral, proceso que originará un volumen importante de puestos de trabajo redundantes.

En todo caso, se trata de un ingreso económico de carácter periódico que el Estado da a cada ciudadano como derecho social. Es una propuesta que surge originalmente en el mundo académico con los trabajos de Van Parjis durante la década de los años 80, en el contexto español se comienza a debatir durante la década de los 90 con las aportaciones de Daniel Raventós Panella (1999) y de José Iglesias Fernández (2002), entre otros, y que a lo largo de las décadas siguientes ha ido ganando espacio político y público. Así mismo, en estos últimos años se han elaborado diversos estudios que abordan la viabilidad técnica y económica de las RB en el territorio español (Pérez, 2015). Así como experiencias piloto, siendo la más destacada la iniciativa puesta en marcha por el gobierno finlandés en el 
periodo 2017-18 para estudiar los efectos de la implantación de un sistema de renta básica en el país ${ }^{3}$.

La RB tiene las siguientes características básicas estructurales (Iglesias Fernández, 2002): Es individual, pues se otorga a la persona y no a la unidad familiar como ocurre con las RMI. Es universal e incondicional abarcando a toda la ciudadanía y se reconoce como un derecho social. Las RMI no son derechos universales pues se dirigen únicamente a colectivos en riesgo social. A su vez no tiene en cuenta la situación y la condición de la persona a la hora de recibir el ingreso. Las RMI realizan una valoración y comprobación de los bienes y situación individual de las personas beneficiarias, dando lugar a la creación de un complejo entramado burocrático y administrativo. Por último, es suficiente para poder cubrir las necesidades sociales y vitales básicas situándose su cuantía para ello por encima del umbral de pobreza. Las RMI no ofrecen una cuantía superior al umbral de pobreza y por ello no permiten dar respuestas a necesidades sociales básicas. Son siguiendo a Antonio Negri (1998), una asalarización de la pobreza, dirigidas a evitar la revuelta social ante el problema estructural de la pobreza.

\subsection{Algunas posibilidades de la renta básica de ciudadanía}

La primera y más obvia de las posibilidades es que la implantación de una RB contribuiría poderosamente a la erradicación de la pobreza derivada de la falta de ingresos (Raventós Panella, 1999). La RB implica una redistribución de la riqueza con mayor alcance que la conseguida por los programas de ingresos mínimos, puesto que tienen un carácter universal y están sólidamente inspiradas en el apoyo mutuo y en una solidaridad fuerte. Es una propuesta dirigida al conjunto de la ciudadanía, no es una medida dirigida a las personas pobres, es universal y no es estigmatizadora.

La segunda de las posibilidades es que contribuiría a reestablecer el equilibrio de fuerzas entre el mundo del capital y el mundo del trabajo, al fortalecer la posición social de los trabajadores de cara a las negociaciones laborales (Standing, 2013). De esta forma, se potenciaría la mejora de las condiciones laborales y se reduciría la precariedad, al existir un colchón económico que permitiera al trabajador el escoger libremente los puestos de trabajo.

Otro de los objetivos que se relacionan con la implantación de la RB es el fomento de la participación ciudadana y de formas de organización basadas en el cooperativismo (Wright, 2001). A su vez favorece el desarrollo de iniciativas emprendedoras, al garantizar un soporte económico básico que permita el desarrollo de proyectos laborales basados en el cooperativismo y que en el contexto económico actual tienen que afrontar grandes dificultades para su puesta en marcha: incertidumbre en relación a su éxito futuro, su posible rentabilidad o la dificultad para acceder a financiación por parte de las entidades bancarias.

Otras posibilidades relacionadas con la implantación de una Renta Básica es la reducción de la economía sumergida y de la trampa de la pobreza, que generan los programas de ingresos mínimos. La Renta Básica por su carácter incondicional y universal no requiere la comprobación de medios, ni la incompatibilidad con otros ingresos económicos. Mientras que los programas de ingresos mínimos están supeditados a las condiciones s Para más información sobre esta experiencia piloto véase: http://www.kela.fi/web/en/basic-income-objectives-and-
implementation 
económicas de las personas beneficiarias, lo que favorece la aparición de actividades laborales informales ubicadas dentro de la economía sumergida.

Por último, la Renta Básica se puede concebir como un vector más dentro de una compleja estrategia de transformación social, que incluiría diferentes acciones en diversas esferas interrelacionadas pero sin un centro nuclear (participación política, decrecimiento, ecofeminismo, municipalismo, educación para la emancipación...) que pueden contribuir a la transformación social mediante la gestación de un nuevo orden, dentro de las contradicciones, problemáticas y grietas del (viejo) orden social dominante (Holloway, 2010).

\subsection{Implicaciones para la educación}

Desde la plataforma que ofrece la Renta Básica, el mundo de la educación se distancia de los principios del Capital humano por los cuales toda educación se entiende como formación profesional y como un subsistema del tejido productivo que provee las competencias requeridas por éste último. Este tipo de fundamentación teórica se relaciona estrechamente con el desarrollo de metodologías didácticas expositivas, en donde el educador transmite las técnicas/habilidades/competencias profesionales y el alumnado las adquiere de forma pasiva, bien de forma teórica, bien mediante la práctica directa instrumental de tales habilidades profesionales. Esta concepción de la educación delimita rígidamente los roles del educador y del alumnado y separa las dimensiones de la teoría y de la práctica, entendiendo esta última como una mera aplicación instrumental de lo teórico. Estamos ante una concepción técnica del currículum educativo, en la cual las personas beneficiarias de Renta Mínima de Inserción tienen unos déficits y unas carencias diagnosticadas por los profesionales de los servicios sociales que participan en el diseño de las acciones de formación y en los itinerarios de inserción.

$\mathrm{Al}$ romper con la sumisión al mundo empresarial y con la centralidad de la empleabilidad, la educación se abre con mayor facilidad a otro tipo de planteamientos. Planteamientos en los cuales sea posible introducir contenidos de relevancia social y de carácter contrahegemónico que sirvan para desvelar las falsedades y las tergiversaciones de la ideología dominante, que sirvan para analizar la utilidad social y los potenciales efectos sociales de los diferentes puestos de trabajo y que sirvan para fomentar otro tipo de valores sobre los que construir nuestras sociedades, valores diferentes a los pregonados por el pensamiento neoliberal y que se sustenten en el apoyo mutuo y la solidaridad.

La educación en general, y la formación para personas adultas en situación de exclusión social de manera concreta, si quiere estar orientada a la transformación social tiene que incluir contenidos que fomenten la reflexión crítica sobre aquellas cuestiones que afecten directamente a las personas implicadas en la acción educativa, tanto educadores como educandos. Por ejemplo, la formación de carácter profesional que se realiza dentro de los subsidios de las RMI tiene que incluir, si quiere ser verdaderamente contrahegemónica, aspectos como el origen y las consecuencias de la precariedad laboral, sobre la utilidad social de los oficios para los que se está formando, sobre la privatización de la educación y otros servicios públicos, sobre el origen y el papel de la pobreza en nuestras sociedades capitalistas, entre otros aspectos y contenidos que sean relevantes socialmente para las personas participantes y para la propia comunidad donde toma lugar la acción educativa.

En esta línea, es necesario reestablecer el equilibrio entre el valor de uso y el valor de cambio en la educación, desequilibrio que con las teorías del capital humano no hace más 
que: a) devaluar las credenciales formativas (cada vez es más necesario tener más titulaciones, certificados y diplomas para obtener un puesto de trabajo precario), y b) considerar la educación como un mero medio para obtener algo a cambio, perdiendo de referencia los aprendizajes o el disfrute personal que se pueda conseguir y cayendo en la espiral del consumismo educativo (Cascante Fernández, 2018). En ese sentido, la Renta Básica se muestra como un mecanismo especialmente potente a la hora de reducir la hegemonía de las ideas basadas en la empleabilidad y en la mercantilización de la educación.

También las propuestas metodológicas y de organización de la educación toman un carácter diferente desde estos planteamientos, distanciándose de los modelos expositivos en donde unos (el personal educador) diseñan, seleccionan los contenidos y los transmiten, mientras que otros (el alumnado) los reciben y asimilan de forma más o menos pasiva. Se enfatiza las metodologías didácticas basadas en la interacción grupal, en los debates y en los diálogos, en los cuales el alumnado adquiere un rol mucho más activo, tanto en el desarrollo de la acción educativa como en la planificación previa de la misma. Desde este punto de vista, se difumina la separación entre técnicos expertos y alumnado, porque quienes enseñan, aprenden y quienes aprenden, enseñan. Metodologías propias de esta perspectiva son los círculos de lectura y las comunidades de aprendizaje (Flecha, 1990), los centros de interés, los procedimientos de investigación-acción (Kemmis y Carr, 1993) o los círculos de enseñanza dialógica (Freire, 1971). Metodologías que tratan de incluir en su desarrollo tanto el papel de las estructuras sociales, como los propios intereses y valores de las personas participantes mediante la interacción, el debate y el diálogo sobre contenidos y temas de enseñanza de carácter académico y/o profesionalizador.

\section{Conclusiones}

En este artículo se ha discutido cómo las políticas tradicionales de lucha contra la pobreza y las acciones formativas insertas en ellas no consiguen ofrecer respuestas válidas a los problemas sociales existentes en este momento histórico. Peor aún, como muestra Oxfam (2017), las transformaciones que el ideario neoliberal está imponiendo en los estados de bienestar están haciendo que nuestras sociedades sean cada vez más desiguales, menos solidarias y más empobrecidas. Ante esta situación, es urgente buscar discursos y prácticas alternativas que rompan con los planteamientos neoliberales, pero que también supongan un avance frente a los caducos procedimientos de intervención socioeducativa típicos del pensamiento humanista.

La Renta Básica es una propuesta que no se dirige meramente a paliar la pobreza, sino que, partiendo de las contradicciones y problemáticas sociales actuales, busca la transformación social. Bajo esta propuesta la educación rompe con los principios del capital humano, de la privatización y del consumismo educativo, y se abre a otras concepciones y espacios de desarrollo tanto en el plano metodológico, como en el curricular, como en la propia concepción de la relación educador-educando.

Para concluir creemos necesaria plantear la siguiente reflexión, y es que desde el $2011 \mathrm{al}$ 2015 se han dedicado más de 7.000 millones de euros al desarrollo de cursos formativos dentro de las políticas sociales de activación y fomento de la empleabilidad. Ese dinero pudo haberse dedicado a la creación directa de puestos de trabajo con utilidad social y con adecuadas condiciones de integración social o al asentamiento de una sólida cobertura de 
ingresos mínimos para los sectores sociales más precarios. ¿Por qué no se hizo? Porque entonces la educación para pobres dejaría de ser un mecanismo de redención y de castigo para el pobre, y ya no sería una oportunidad de negocio para el sector privado, negocio al que acuden tanto ONG, empresas, fundaciones, asociaciones, entidades sindicales, colegios oficiales, etc. Unas siguiendo principios filantrópicos y humanistas, y otras siguiendo principios mercantilistas. Pero en todo caso, todas ellas acudiendo juntas a la llamada del negocio de la pobreza.

\section{Referencias}

Althusser, L. (2004). Ideología y aparatos ideológicos del estado. En S. Zizek (Comp.), Ideología. Un mapa de la cuestión. Buenos Aires: Fondo de Cultura Económica.

Apple, M. (2002). Educando como dios manda: Mercado, niveles, religión y desigualdad. Barcelona: Paidós.

Apple, M. (2013). Can education change society? Londres: Routledge.

Arriba González de Durana, A. (2014). El papel de la garantía de ingresos mínimos frente a la crisis. Madrid: Fundación FOESSA.

Avent, R. (2017). La riqueza de los humanos: El trabajo en el siglo XXI. Madrid: Alianza.

Ball, S. (2012). Global education. New policy networks and the neo-liberal imaginary. Londres: Routledge.

Bauman, Z. (2004). Vidas desperdiciadas. La modernidad y sus parias. Barcelona: Paidós.

Becker, G. (1985). El capital humano. Madrid: Alianza.

Bowles, S. y Gintis, H. (1983). El problema de la teoría del capital humano. Una crítica marxista. En L. Toharia (Comp.), El mercado de trabajo: Teorías y aplicaciones. Madrid: Alianza.

Buenfil Burgos, R. (1996). Foucault y la analítica del discurso. Topos y Tropos, 2, 1-12.

Cascante Fernández, C. (2018, julio). Education as a common good use. Value and exchange value of educational goods. Comunicación presentada en VIII International Conference on Critical Education. University of East London, Londres.

Chang, H. (2012). 23 cosas que no te cuentan sobre el capitalismo. Madrid: Debate.

Comisión Europea. (2010). Europa 2020. Una estrategia para un crecimiento inteligente, sostenible e integrador. Bruselas: Comisión Europea.

Díez Gutiérrez, E. J. (2007). La globalización neoliberal y sus repercusiones en la educación. Barcelona: El Roure.

Esping-Andersen, G. (2000). Los fundamentos sociales de las economías postindustriales. Barcelona: Ariel.

EUROSTAT. (2018). People at risk of poverty or social exclusion. Recuperado de http:/ / ec.europa.eu/eurostat $/ \mathrm{tgm} /$ table.do? tab=table\&plugin $=1 \&$ language $=$ en $\&$ pcode $=\mathrm{t}$ 2020_50

Fernández, G., Martínez Virto, L., Pérez, J., Pérez, B. y Sánchez, E. (2014). Qué hacemos para construir una sociedad más igualitaria y contra el aumento de la exclusión social y la pobreza. Madrid: Akal.

Flecha, R. (1990). Educación de las personas adultas. Propuestas para los noventa. Barcelona: Roure.

Foucault, M. (2009). El nacimiento de la biopolítica. Madrid: Akal. 
Frazer, H. y Marlier, E. (2016). Minimum income schemes in Europe. A study of nacional policies. Bruselas: Comisión Europea.

Freire, P. (1971). Pedagogía del oprimido. Madrid: Siglo XXI.

Gómez Sánchez, L., Navas Saurín, A. y Bernard García, J. (2005). Sujetos flexibles: Racionalidades neoliberales y políticas educativas. Archivos Analíticos de Políticas Educativas, 13, 44-67.

Harvey, D. (2012). The enigma of capital: And the crises of capitalism. Oxford: University Press.

Harvey, D. (2013). Seventeen contradictions and the end of capitalism. Oxford: University Press.

Hill, D. (2013). Class struggle and education. Neoliberalism, (neo)-conservatism, and the capitalist assault on public education. Critical Education, 10(4), 23-54.

Holloway, J. (2010). Agrietar el capitalismo. El hacer contra el trabajo. Barcelona: El viejo topo.

Iglesias Fernández, J. (2002). Las rentas básicas. El modelo fuerte de implantación territorial, Barcelona: El Viejo Topo.

Kemmis, S. y Carr, W. (1986). Teoría crítica de la enseñanza. La investigación-acción en la formación del profesorado. Barcelona: Martínez Roca.

Krugman, P. (24 de septiembre de 2012). Desdén por los trabajadores. El País. Recuperado de https://elpais.com/internacional/2012/o9/23/actualidad/1348418999_156122.html

Laclau, E. y Mouffe, C. (1987). Estrategía y hegemonía socialista. Radicalización de la democracia. Ciudad de México: Fondo de Cultura.

Malgesini, G. (2017). Developments in relation to minimum income schemes. Recuperado de https://eminnetwork.files.wordpress.com/2013/04/spain-minimum-income-update2017.pdf

Marshall, T. H. (1950). Citizenship and social class and other essays. Cambridge: Cambridge University Press.

Ministerio de Servicios Sociales. (2015). Informe de rentas mínimas de inserción. Año 2015. Recuperado de https://www.msssi.gob.es/

Muñoz de Bustillo, R. y Bonete, R. (2009). Introducción a la UE: Un análisis desde la economía. Madrid: Alianza.

Negri, A. (1998): El exilio. Barcelona: El Viejo Topo.

Oliver, P. (2013). Burorrepresión. Sanción administrativa y control social. Albacete: Bomarzo.

Oxfam. (2017). An economy for the 99\%. Recuperado de https://d1 tn3vj7xz9fdh.cloudfront.net/s3fspublic/file_attachments/bp-economy-for-99-percent-160117-en.pdf

Pérez, C. (2015). Renta básica universal. La peor de la soluciones (a excepción de todas las demás). Madrid: Clave intelectual.

Piketty, T. (2014). El capital en el siglo XXI. Madrid: Fondo de Cultura Económica.

Pinker, S. (2018). En defensa de la ilustración Por la razón, la ciencia, el humanismo y el progreso. Barcelona: Paidós.

Raventós Panella, D. (1999). El derecho a la existencia. Barcelona: Ariel.

Rodríguez Fernández, J. R. (2013). Las políticas de lucha contra la exclusión social y su relación con la pedagogía social. Los programas de rentas mínimas de inserción y la propuesta de las rentas básicas. Archivos Analíticos de Políticas Educativas, 21(40), 43-68. https://doi.org/10.14507/epaa.v2 1n40.2013 
Rodríguez Fernández, J. R. (2016). Entreteniendo a los pobres. Una crítica político-ideológica de las medidas de lucha contra la exclusión social. Albacete: Bomarzo.

Schön, D. (1992). La formación de profesionales reflexivos. Hacia un nuevo diseño de la enseñanza y el aprendizaje de las profesionales. Barcelona: Paidós.

Schultz, T. W. (1962). Investment in human beings. Chicago, IL: University of Chicago Press.

Standing, G. (2013). El precariado: Una nueva clase social. Barcelona: Pasado \& Presente.

Wacquant, L. (2010). Castigar a los pobres. El gobierno neoliberal de la inseguridad social. Barcelona: Gedisa.

Whitty, G., Power, S. y Halpin, D. (1999). La escuela, el estado y el mercado. Madrid: Morata.

Wright, E. O. (2001). Propuestas utópicas reales para reducir la desigualdad de ingresos y riqueza. En R. Gargarella y F. Ovejero (Comps.), Razones para el socialismo (pp. 97-125). Barcelona: Paidós.

Wrigley, T. (2005). Blair's vision for education: Business, business, business. International Socialism, $106,35-57$.

\section{Breve CV de los autores}

\section{Juan Ramón Rodríguez Fernández}

Licenciado y doctor en Pedagogía (2015) por la Universidad de Oviedo. He realizado estancias académicas en las siguientes instituciones: State University of New York (USA), University of Essex y Anglia Ruskin University (UK). Ha trabajado como educador y pedagogo en diversos centros de servicios sociales en la Consejería de Bienestar Social de Asturias. Actualmente es pedagogo en el Instituto Asturiano de la Administración Pública "Adolfo Posada" y profesor asociado en el Grado de Educación Social de la Facultad de Educación de la Universidad de León. Tiene publicaciones en revistas especializadas y ha participado en varios congresos internacionales de Educación y Pedagogia. Autor del libro "Entreteniendo a los pobres. Una crítica político ideológica de las medidas de lucha contra la pobreza" (2016). Editorial Bomarzo. ORCID ID: https://orcid.org/0000-0003-25230681.Email: jrodrf@unileon.es

\section{Enrique Javier Díez Gutiérrez}

Doctor en Ciencias de la Educación. Licenciado en Filosofía. Diplomado en Trabajo Social y Educación Social. Profesor de la Facultad de Educación de la Universidad de León. Proyectos de investigación I+D: 2015-2017: «Ecologías del aprendizaje en contextos múltiples», MINECO. Referencia: EDU2014-51961-P. Publicaciones: Neoliberalismo educativo (Octaedro, 2018), La educación que necesitamos (Akal, 2016), Qué hacemos con la Universidad (Akal, 2014), Qué hacemos con la educación (Akal, 2012), Educación Intercultural (Aljibe, 2012), "Decrecimiento y educación" en Decrecimientos (Catarata, 2011), La Memoria Histórica en los libros de texto (2012), Globalización y Educación Crítica publicado en Colombia (Desde Abajo, 2009). ORCID ID: https://orcid.org/Ooooooo3-3399-5318. Email: ejdieg@unileon.es 